\title{
Diagnosing particle acceleration in relativistic jets
}

\author{
Markus Böttcher ${ }^{1}$, Matthew G. Baring ${ }^{2}$, Edison P. Liang ${ }^{2}$, Errol J. \\ Summerlin ${ }^{3}$, Wen Fu ${ }^{2}$, Ian A. Smith ${ }^{2}$ and Parisa Roustazadeh ${ }^{2}$ \\ ${ }^{1}$ Centre for Space Research, North-West University \\ Potchefstroom, 2520, South Africa \\ email: Markus. Bottcher@nwu.ac.za \\ ${ }^{2}$ Department of Physics and Astronomy, Rice University \\ MS 108, 6100 Main Street, Houston, TX 77005, USA \\ ${ }^{3}$ Heliospheric Physics Laboratory, Code 672 \\ NASA Goddard Space Flight Center, Greenbelt, MD 20770, USA
}

\begin{abstract}
The high-energy emission from blazars and other relativistic jet sources indicates that electrons are accelerated to ultra-relativistic $(\mathrm{GeV}-\mathrm{TeV})$ energies in these systems. This paper summarizes recent results from numerical studies of two fundamentally different particle acceleration mechanisms potentially at work in relativistic jets: Magnetic-field generation and relativistic particle acceleration in relativistic shear layers, which are likely to be present in relativistic jets, is studied via Particle-in-Cell (PIC) simulations. Diffusive shock acceleration at relativistic shocks is investigated using Monte-Carlo simulations. The resulting magneticfield configurations and thermal + non-thermal particle distributions are then used to predict multi-wavelength radiative (synchrotron + Compton) signatures of both acceleration scenarios. In particular, we address how anisotropic shear-layer acceleration may be able to circumvent the well-known Lorentz-factor crisis, and how the self-consistent evaluation of thermal + nonthermal particle populations in diffusive shock acceleration simulations provides tests of the bulk Comptonization model for the Big Blue Bump observed in the SEDs of several blazars.
\end{abstract}

Keywords. acceleration of particles, radiation mechanisms: nonthermal, BL Lacertae objects: individual (AO 0235+164, Mrk 501), gamma rays: theory

\section{Introduction}

The relativistic jets of active galactic nuclei (AGN) and gamma-ray bursts (GRBs) are well-established sites of ultrarelativistic particle acceleration, as evidenced by their prominent $\gamma$-ray emission. In spite of many decades of intensive efforts, the nature of the emitting relativistic particles and the mode of acceleration of particles to $\mathrm{GeV}-\mathrm{TeV}$ energies remain poorly understood. Potential modes of particle acceleration include diffusive shock acceleration (DSA) at relativistic shocks (e.g., Drury (1983), Jones \& Ellison (1991), Niemiec \& Ostrowski (2004), Summerlin \& Baring (2012)), particle acceleration at relativistic shear layers (e.g., Berezhko (1981), Stawarz \& Ostrowski (2002), Rieger \& Duffy (2006), Liang et al. (2013a), Liang et al. (2013b)), and magnetic reconnection (e.g., Romanova \& Lovelace (1992), Giannios et al. (2009), De Gouveia Dal Pino et al. (2010), Sironi \& Spitkovsky (2014)). This paper provides a brief summary of recent results obtained by our group, concerning the radiative signatures of relativistic shock acceleration in blazar jets, and PIC simulations of magnetic-field generation and particle acceleration in relativistic shear layers. 

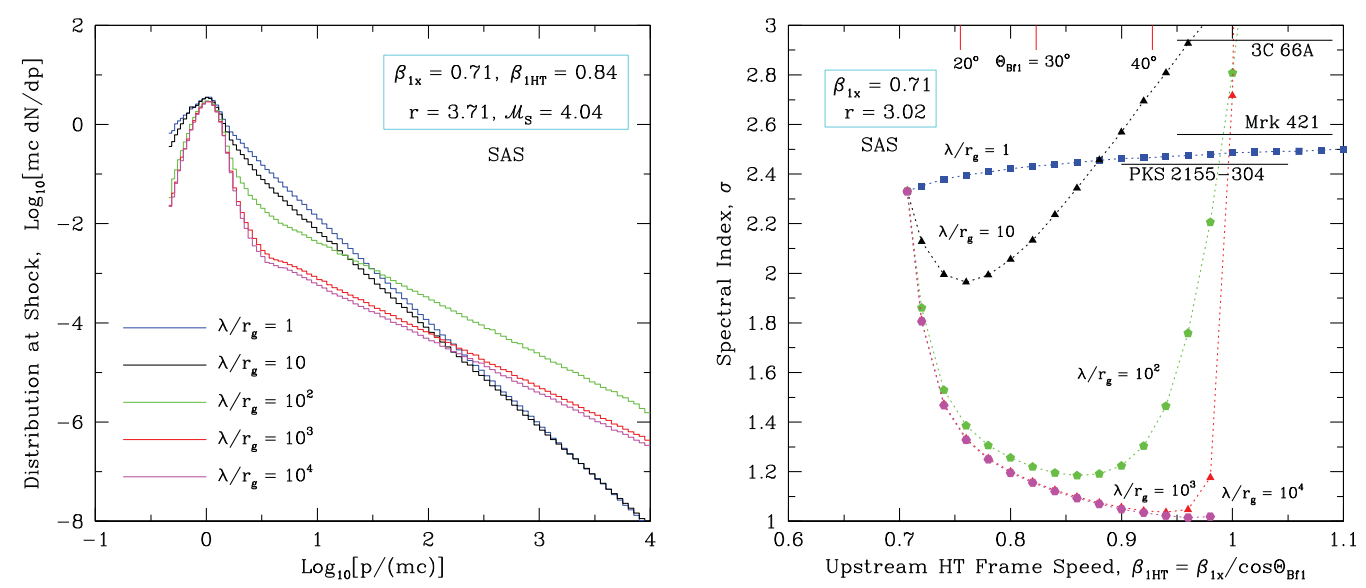

Figure 1. Left: Thermal + non-thermal particle distributions resulting from DSA at a relativistic, sub-luminal shock, for various values of $\eta=\lambda / r_{g} \equiv$ const. (i.e., $\alpha=1$ ). Right: Dependence of the non-thermal spectral index $\sigma$ (where the non-thermal tail of the particle distribution is $n(\gamma) \propto \gamma^{-\sigma}$ ) on the shock obliquity, for various values of $\eta$ (from Summerlin \& Baring (2012)).

\section{Relativistic shocks}

Diffusive shock acceleration (DSA) has long been considered one of the principal mechanisms to accelerate particles to $\mathrm{GeV}-\mathrm{TeV}$ energies in relativistic jets. It is very efficient and very fast. The characteristic acceleration time scale of DSA is of the order of the gyro time scale, $t_{\text {gyr }}=\gamma m c /(e B)$, where $\gamma$ is the particle's Lorentz factor, since diffusive collisions of electrons and ions with MHD turbulence, which are the fundamental mechanism facilitating the reflection of particles from the downstream to the upstream plasma and vice versa, are usually dominated by gyroresonant interactions.

The pitch-angle scattering mean free path $\lambda$ is then at least comparable to the gyro radius, $r_{g}=p c /(e B)=\gamma \beta m c^{2} /(e B)$. The limiting case $\lambda \sim r_{g}$ is known as the Bohm diffusion limit. More generally, it is common to parameterize the mean free path as $\lambda=\eta r_{g}$, where $\eta$ is a free parameter, which may be momentum-dependent. Following Summerlin \& Baring (2012), we define $\eta(p) \equiv \eta_{1}\left(p / p_{1}\right)^{\alpha-1}$ so that $\lambda=\lambda_{1}\left(p / p_{1}\right)^{\alpha}$. Summerlin \& Baring (2012) have demonstrated that both the fraction of particles that are accelerated out of the thermal pool into a non-thermal, power-law tail of ultrarelativistic particles, and the spectral index $\sigma$ of the non-thermal power-law tail depend sensitively on both the mean-free path parameter $\eta$ and the shock obliquity (see Figure 1).

Thermal + non-thermal particle distributions generated with the Monte-Carlo code by Summerlin \& Baring (2012) have been used to evaluate the radiative (synchrotron + Compton) output from various shock acceleration scenarios. For this purpose, the radiation transfer code of Böttcher et al. (1997), Böttcher \& Chiang (2002), Böttcher et al. (2013) has been adapted to use the Monte-Carlo generated particle distributions as input. Using an iterative scheme, the input particle distributions were evolved towards an equilibrium between particle injection (using the Monte-Carlo input), radiative cooling, and escape from the shock environment, taking into account self-consistently synchrotron, synchrotron self-Compton and external-Compton radiation. The high-energy cut-off of the non-thermal particle distribution is determined self-consistently by the balance between particle acceleration and radiative cooling, where the acceleration time scale is taken as $t_{\text {acc }}(p)=\eta(p) t_{\text {gyr }}$.

Figure 2 illustrates the resulting fits to the multi-wavelength spectral energy distributions (SEDs) of two Fermi-LAT detected $\gamma$-ray blazars: The low-frequency-peaked BL 

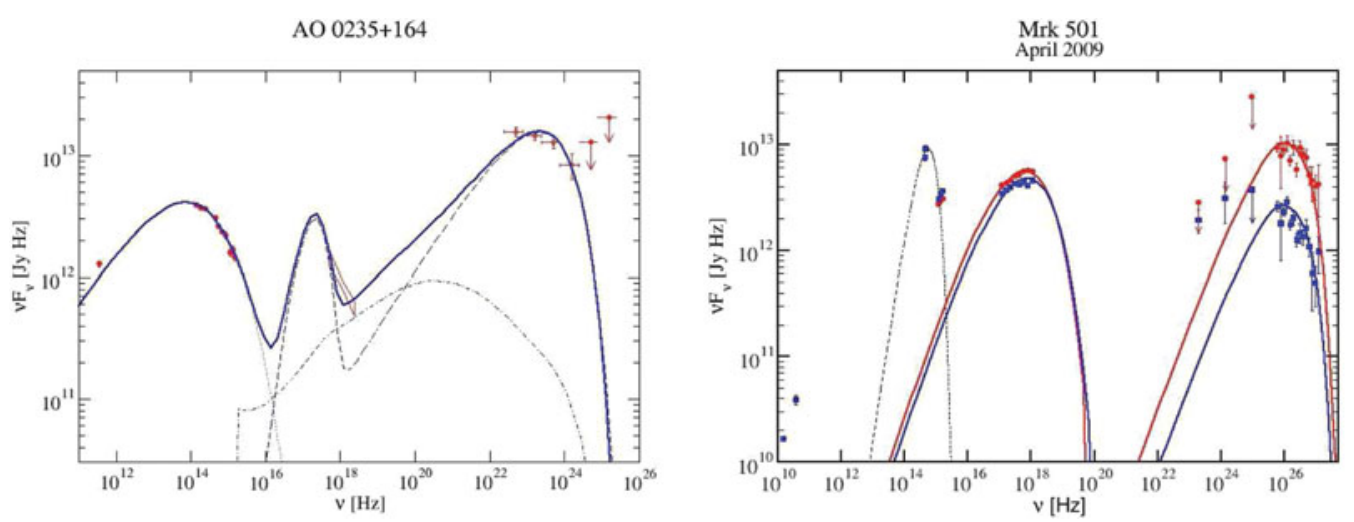

Figure 2. Left: Multi-wavelength SED and fit with our shock-acceleration - radiation simulations. The fit requires a strongly momentum-dependent mean-free path parameter with $\alpha=3$. The thermal population in the jet produces a pronounced external-Compton bump due to Bulk Compton Emission (Sikora et al. (1997)). Data from Ackermann et al. (2012). Right: Fit to two SEDs of Mrk 501 in 2009. Here, a weaker momentum dependence of $\alpha=1.5$ is sufficient to produce an acceptable fit.

Lac object AO 0235+164 (Ackermann et al. (2012)) and the high-frequency peaked BL Lac object Mrk 501 (Aliu et al., in prep.). The fitting procedure revealed a fundamental requirement on the DSA parameters: As evident from Figure 1 (left), a moderately small value of $\eta$ is required at low particle momenta in order to achieve efficient injection of particles into the non-thermal tail. However, in order to achieve a moderate value of $\gamma_{\max } \sim 10^{4}(\mathrm{AO} 0235+164)$ to $10^{6}$ (Mrk 501), as required by the fits for reasonable values of the magnetic field of $B \sim 0.1$ - a few Gauss (corresponding to a magnetization parameter of $\left.u_{B} / u_{e} \sim 0.1\right)$, values of $\eta\left(\gamma_{\max }\right) \sim 10^{8}(\mathrm{AO} 0235+164)$ and $10^{4}$ (Mrk 501) are required, implying a strong momentum-dependence of the pitch-angle mean-free path parameter, with $\alpha=3$ for AO $0235+164$ and $\alpha=1.5$ for Mrk 501, respectively. Other parameters of the fits are in the standard range usually adopted for SED fits to these blazars.

An interesting aspect of the fit to AO $0235+164$ in Figure 2 (left) is the emergence of the Bulk Compton Signature, suggested by Sikora et al. (1997) to be responsible for a soft X-ray excess in the SEDs of blazars, as observed in this example. This feature provides a stringent constraint on the value of $\eta$ at low energies, which determines the relative normalization of the thermal to the non-thermal particle populations.

\section{Relativistic shear layers}

There is mounting evidence, both direct observational (e.g., Giroletti et al. (2004)) and from MHD simulations (e.g., Meliani \& Keppens (2007), Mizuno et al. (2007)) that the relativistic jets of AGN maintain a radially stratified structure with a fast, inner spine of low-density material, surrounded by a denser, slower, outer sheath. The strong velocity shears necessarily present in such a configuration, provide a promising avenue for relativistic particle acceleration (e.g., Berezhko (1981), Stawarz \& Ostrowski (2002), Rieger \& Duffy (2006)) as such shears are likely to be dissipative due to the KelvinHelmholtz instability (Chandrasekhar (1981)).

In recent work (Liang et al. (2013a), Liang et al. (2013b)), we have conducted PIC simulations of relativistic shear layers to investigate the self-generation of magnetic and electric fields and relativistic particle acceleration, for pure electron-positron, 

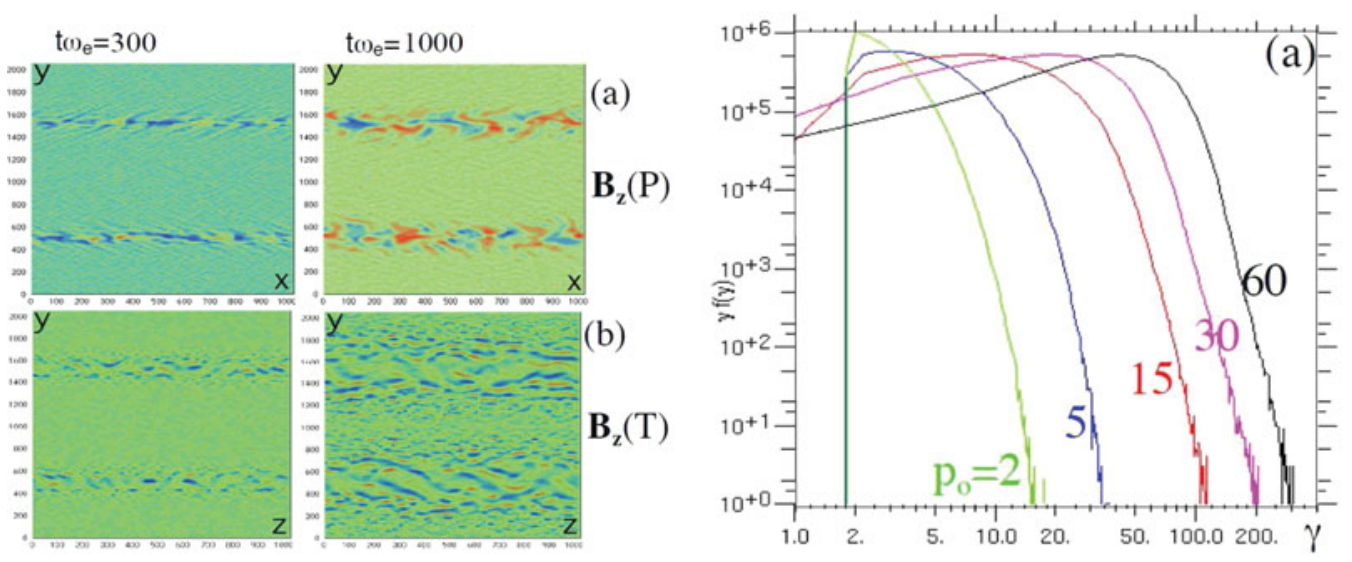

Figure 3. Left: Self-generated magnetic fields $B_{z}$ (in the plane of the shear layer, perpendicular to the shear flow) in a pure electron-positron pair plasma with $p_{0}=15$ for simulations in the shear momentum $(x-y)$ plane (P modes, upper panels) and in the transverse $(y-z)$ plane ( $\mathrm{T}$ modes, lower panels). Right: Angle-averaged particle distributions in the vicinity of the shear layers for various values of the shear velocity $p_{0}$, for a pure $e^{-} e^{+}$pair plasma. (From Liang et al. (2013a).)

electron-proton, and hybrid electron-positron-proton plasmas. We used the 2.5-dimensional PIC code ZOHAR II, available at LLNL. The simulations are performed in the centerof-momentum frame of the shear, in which the spine and sheath are counter-streaming with identical velocities, parameterized through $p_{0} \equiv \beta \gamma$ where $\beta=v / c$ is the normalized bulk velocity and $\gamma$ the corresponding Lorentz factor. The $x$ axis is defined along the direction of the flow, the $y$ direction is perpendicular to the shear layers. Both spine and sheath are initially unmagnetized. Figure 3 (left) illustrates the development of selfgenerated magnetic fields $B_{z}$ (i.e., in the plane of the shear layer, perpendicular to the shear flow), which is the dominant magnetic-field component, for a pure electron-positron pair plasma. It can be seen that turbulent magnetic fields with a strength of a few $\%$ of equipartition with the kinetic energy density in the plasma are being self-generated by energy dissipation in the shear layer.

The right panel of Figure 3 shows the resulting angle-averaged particle distributions in the vicinity of the shear layer, for various values of the shear velocity $p_{0}$. The distributions assume a quasi-Maxwellian shape peaking at $\gamma_{p} \sim p_{0} / 2$, and a power-law tail emerging for $p_{0} \gtrsim 15$. A plot of the angle-dependent particle momenta in this distribution shows that particles are being accelerated primarily across the shear layer (the $y$ direction), i.e., perpendicular to the self-generated magnetic fields. They are therefore expected to emit synchrotron radiation efficiently, and anisotropically, reflecting the anisotropy of the particle distribution.

Figure 4 illustrates that the results for pure $e^{-} p$ plasmas and for hybrid $e^{+} e^{-} p$ plasmas are drastically different from the pure $e^{-} e^{+}$case. The left panel shows the magnetic field $B_{z}$ (in the shear layer, perpendicular to the flow) and electric field $E_{y}$ (perpendicular to the shear layer), which are the dominant field components, after $t \omega_{\mathrm{e}}=3000$, where $\omega_{\mathrm{e}}$ is the electron plasma frequency, for a pure $e^{-} p$ plasma (top), a hybrid plasma with $90 \%$ $e^{-} p$ and $10 \% e^{-} e^{+}$plasma (middle) and a pure $e^{-} e^{+}$plasma (bottom). One can see that, due to the much larger inertia of the protons, compared to electrons, the pure $e^{-} p$ plasma produces stable layers of self-generated electric and magnetic fields, and the stable electric fields are very efficient in accelerating electrons across the shear layers. The right panel shows the resulting angle-averaged electron distributions. The top-right panel of Figure 4 

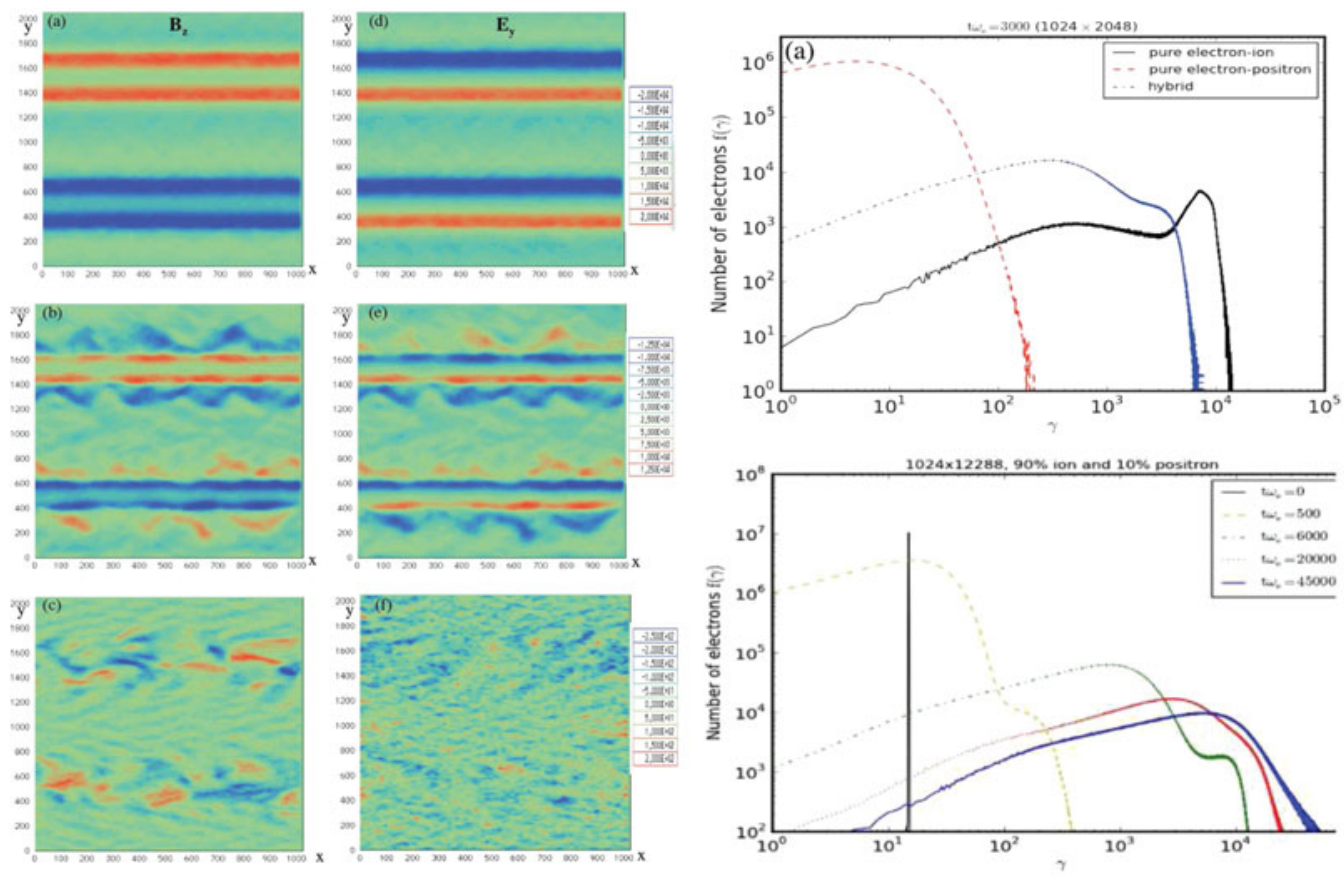

Figure 4. Left: Self-generated magnetic fields $B_{z}$ (in the plane of the shear layer, perpendicular to the flow) and electric fields $E_{y}$ (perpendicular to the shear layer), for a pure $e^{-} p$ plasma (top), a hybrid plasma of $90 \% e^{-} p$ and $10 \% e^{-} e^{+}$(middle) and a pure pair plasma (bottom) after $t \omega_{e}=3000$ for $p_{0}=15$. Top right: Angle-averaged particle distributions for the three simulations illustrated in the left panel. Bottom right: Time evolution of the electron distribution for the hybrid plasma case. (From Liang et al. (2013b).)

illustrates that the pure $e^{-} p$ case produces an electron spectrum that resembles a double Maxwellian, with a high-energy component peaking at $\gamma_{p} \sim p_{0} m_{p} /\left(2 m_{e}\right)$. It is only the hybrid case which produces a relativistic quasi-Maxwellian and an extended high-energy tail of ultrarelativistic particles, which is required to reproduce the non-thermal $\gamma$-ray spectra observed in relativistic jet sources, such as blazars and gamma-ray bursts. The bottom-right panel of Figure 4 shows the time evolution of the electron distribution in the hybrid case and illustrates how gradually, a $n(\gamma) \propto \gamma^{-3}$ power-law tail of ultrarelativistic electrons develops.

\section{Summary and Conclusions}

We have summarized recent results of numerical simulations of particle acceleration at relativistic, oblique shocks and relativistic shear layers, with particular emphasis on applications to the relativistic jets of blazars and gamma-ray bursts. Relativistic particle spectra produced through diffusive shock acceleration were used to predict self-consistently the emerging SEDs from synchrotron, synchrotron self-Compton and external-Compton radiation. These were used to produce fits to the SEDs of the BL Lac objects AO 0235+164 and Mrk 501. In both cases, we found that such fits require a strongly momentumdependent pitch-angle scattering mean free path, with $\lambda \propto p^{3}$ for AO $0235+164$ and $\lambda \propto p^{1.5}$ for Mrk 501, respectively. Such a momentum dependence is naturally expected in situations where the turbulence responsible for the diffusive pitch-angle scattering is produced on large scales (the "stirring scale"), and then cascades down to smaller scales. 
The resulting plasma wave spectrum is expected to level off at long wavelengths, i.e., the regime with which the highest-energy particles interact, leading to an increasing mean free path. A detailed discussion of the implications of a strongly momentum-dependent mean free path in relation to magnetic-field turbulence is presented in Baring et al. (2014).

Our spectral fit to AO $0235+164$ also provided the first self-consistent evaluation of the bulk Compton process on the thermal particle population, where the relative normalization of the thermal to non-thermal particle populations (and, hence, of the bulk Compton emission to the non-thermal synchrotron and Compton emissions) is a result of the diffusive-shock-acceleration Monte-Carlo simulations.

The self-generation of magnetic and electric fields in initially unmagnetized relativistic shear flows has been studied using PIC simulations. The SEDs of blazars and GRBs indicate the presence of non-thermal power-law distributions of relativistic particles. Our simulations have shown that such distributions result only in the case of hybrid electronpositron-proton plasmas. The relativistic electron distributions are highly anisotropic, with particle acceleration happening primarily perpendicular to the shear layer by selfgenerated electric fields. As the self-generated magnetic fields are produced primarily in the plane of the shear layer, perpendicular to the flow, the relativistic electrons are expected to radiate efficiently, and anisotropically, through synchrotron and Compton emission. This provides additional beaming of the non-thermal radiation produced in relativistic jets, on top of the standard Doppler boosting resulting from the relativistic bulk motion. This may provide a promising way to solve the "Lorentz factor crisis" in the relativistic jets of blazars (Lyutikov \& Lister (2010)).

\section{References}

Ackermann, M., et al. 2012, ApJ, 751, 159

Baring, M. G., Böttcher, M., \& Summerlin, E. J. 2014, Proc. of "High Energy Phenomena in Relativistic Outflows (HEPRO IV)", F. A. Aharonian, et al. (Eds.), IJMP Conf Ser. 28, 1460167

Berezhko, E. G. 1981, JETPL, 33, 399

Böttcher, M., Mause, H., \& Schlickeiser, R. 1997, A\&A, 324, 395

Böttcher, M. \& Chiang, J. 2002, ApJ, 581, 127

Böttcher, M., Reimer, A., Sweeney, K., \& Prakash, A. 2013, ApJ, 768, 54

Chandrasekhar, S. 1981 Hydrodynamic and Hydromagnetic Stability, New York: Dover

De Gouveia Dal Pino, E. M., Piovezan, P. P., \& Kadowaki, L. H. S. 2010, Aש\&A, 518, A5

Drury, L. O'C 1983, Rep. Prog. Phys., 46, 973

Giannios, D., Uzdensky, D. A., \& Begelman, M. C. 2009, MNRAS, 395, L29

Giroletti, M., et al. 2004, ApJ, 600, 127

Jones, F. C. \& Ellison, D. C. 1991, Space Sci. Rev., 58, 259

Liang, E. P., Böttcher, M., \& Smith, I. A. 2013a, ApJ, 766, L19

Liang, E. P., Fu, W., Böttcher, M., Smith, I. A., \& Roustazadeh, P. 2013b, ApJ, 779, L27

Lyutikov, M. \& Lister, M. 2010, ApJ, 722, 197

Meliani, Z. \& Keppens, R. 2007, A\&BA, 475, 785

Mizuno, Y., Hardee, P., \& Nishikawa, K. I. 2007, ApJ, 662, 835

Niemiec, J. \& Ostrowski, M. 2004, ApJ, 610, 851

Rieger, F. \& Duffy, P. 2006, ApJ, 652, 1044

Romanova, M. M. \& Lovelace, R. V. E. 1992, A\& $A, 262,26$

Sikora, M., et al. 1997, ApJ, 484, 108

Sironi, L. \& Spitkovsky, A. 2014, ApJ, 783, L21

Stawarz, L. \& Ostrowski, M. 2002, ApJ, 578, 763

Summerlin, E. J. \& Baring, M. G. 2012, ApJ, 745, 63 